\title{
Spontaneous spatial information provided by dementia patients and elderly controls in narratives
}

\begin{abstract}
Solveig Bosse*
Abstract. Patients diagnosed with dementia of the Alzheimer's type (DAT) often show two symptoms early on: the inability to navigate space effectively and a deterioration of their language skills, especially on semantic tasks. In this work, I look at whether the inability to navigate space is reflected in the spontaneous speech of DAT patients. Through a corpus analysis of narratives by DAT and control participants, I investigate the hypothesis that DAT patients provide less spatial information than healthy controls (mirroring the decline of effective spatial reasoning in language production). This hypothesis was not supported for locative/stative descriptions using in, on, and at; both groups included this information equally often. However, significant differences between the groups were found for the inclusion of the spatial terms left and right as well directional/dynamic spatial information indicated by into, onto, from, and to. This difference between locative/stative and directional/dynamic spatial information has not previously been reported. I argue that it aligns with Chatterjee's (2008) proposal of the relational features of spatial language and that these features can be differently affected in DAT patients, aligning it with the spatial navigation impairment in these participants.
\end{abstract}

Keywords. spatial language; Alzheimer's disease; direction; location

1. Introduction. Two early signs of dementia, in particular Alzheimer's Disease (AD), are that the person fails to navigate space effectively - the person gets lost on their way (see among others Cherrier et al., 2001, and references cited therein) - and that the person shows symptoms of linguistic deterioration (see among others Verma \& Howard, 2012, and references cited therein). In this paper, I investigate whether people with a diagnosis of probable dementia of the Alzheimer's type (DAT)' show a connection between these two hallmarks of DAT by investigating whether DAT participants spontaneously include the same amount of spatial information in a narrative as (elderly) control subjects do. The investigated hypothesis is that DAT participants include less spatial information in their narratives than the control group (reflecting the deficits in spatial cognition in language). This work is a first step in investigating whether the deterioration of the two cognitive systems (spatial navigation and language) in DAT patients are linked or independent of each other.

The article is structured as follows: in section 2, I provide the background on spatial terms in English (section 2.1) as well as spatial reasoning (section 2.2) and language skills (section 2.3) in DAT patients. Following that (section 3), I describe the rational for my study, its materials and participants. In section 4, I present the results of my work and discuss the findings (section 5). The paper concludes in section 6 .

\footnotetext{
${ }^{*}$ Many thanks to Mark Johnson and the Friday Morning Writing Group for help with this paper. All mistakes are mine. Much of the work was done while at East Carolina University. Author: Solveig Bosse, Brain Capital (Solveig.Bosse@gmail.com)

${ }^{1}$ A variety of different abbreviations are used in the literature for people with a diagnosis of (possible/probable) Alzheimer's disease. For consistency purposes, I will refer to the disease as $D A T$ and the affected people as DAT patients or DAT participants throughout this paper.
} 


\section{Background.}

2.1 TALKING ABOUT SPACE. In English, much spatial information is conveyed by prepositions (e.g. in, on, toward, over, under, between). These prepositions can be used to describe stative locative arrangements, such as entity $X$ is on/in/ under entity $Y$, as well as dynamic movements through space, such as entity $X$ moves towards/ into/ from entity $Y$. In addition, verbs can carry certain types of spatial information (e.g. enter, exit, ascend, stand/lay). Lastly, the cardinal coordinates (north, south, east, west) as well as the egocentric terms of right and left can provide spatial information.

Many studies have investigated the influences of a language's spatial terminology in relation to the native speakers' spatial reasoning and memory as well as the acquisition of these terms and the development of spatial reasoning in children (see Hermer-Vazquez et al., 2001; Munnich et al., 2001 and references cited therein). There is currently no consensus on what effects spatial language has on other cognitive tasks (spatial or non-spatial). One theory seems to be that language is a cognitive system that can be temporarily recruited to solve spatial problems but is neither necessary nor sufficient for such tasks (Landau et al., 2009). The role of language in thinking and potential influences of language (and its availability) on thought are unclear at this point (Tillas, 2015). My research follows the call for detailed studies of spatial language by Chatterjee (2008, p. 234):

"Understanding profound deficits of thought and language experienced by braindamaged subjects is critical to designing targeted rehabilitation interventions.

Ultimately, we need to know the nature of communication deficits experienced by aphasic individuals beyond simply their naming deficits. A greater focus on spatial language and its deficits will deepen our understanding of the communication problems aphasic individuals might experience that would not be evident when simply testing for object knowledge or even syntactic competence."

2.2 DAT AND SPATIAL REASONING. DAT patients and their ability to navigate space, orient themselves in space, perform mental rotation task have often been investigated. Some results relevant for my work are described in the following.

In navigation tasks, the participant is usually asked to navigate a route either on a 2D map, physically towards a particular goal or along a predetermined path, or in a computer-supported virtual reality. There is overall consensus that spatial navigation is difficult for DAT patients and that their spatial skills deteriorate over the course of the disease to the point that patients get lost even in familiar environments such as their own homes (Coughlan et al., 2018, among others).

These spatial navigation experiments often investigate both egocentric and allocentric navigation strategies (see Coughlan et al., 2018, among many others for descriptions). The former are strategies where someone evaluates the spatial scene in relation to their own body. This strategy is usually used for navigating the same path repeatedly. In allocentric tasks, the relation between different objects or landmarks are used for navigational purposes, often to navigate an unknown space. Healthy humans employ both strategies together to navigate space successfully. Brouwers et al. (1984) show that DAT patients perform on par with control subjects

\footnotetext{
${ }^{2}$ Kemmerer (2005) shows that despite the fact that most of the English spatial prepositions also have a temporal use, the two uses can be selectively impaired. He consequently argues that while historically the connection between the two interpretations might have been metaphorical, this is not how current English speakers process the two uses of these prepositions; rather, the spatial and the temporal interpretations of the preposition are two independent processes.
} 
on spatial tasks that involve an egocentric space but not on extrapersonal/allocentric tasks. This is in contrast to Flicker et al. (1988) who report that left-right orientation is impaired in DAT patients while no such impairment was observed for the control group of older adults. Recently, researchers seem to find increased evidence that spatial navigational skills decline with age, with the observed spatial/topographical disorientation being significantly worse in DAT patients (see among others Lithfous et al., 2013; Coughlan et al., 2018). DAT patients perform worse than controls on real-life navigation tasks that including route learning, drawing of the route, and recognizing the same route (Cherrier, et al., 2001; deIpolyi et. al., 2007). Furthermore, deIpolyi et al. (2007. p. 990) argue that the "... navigation impairments are not a product of generalized cognitive decay and instead reflect selective deficits in the spatial domain." This impairment in spatial navigation is so unique in its severity that Coughlan et al. (2018) argue for it to be integrated better into the DAT diagnostics. However, the DAT patients performed equally well as the controls in recognizing landmarks that had been pointed out to them on the route in these studies (Cherrier, et al., 2001; deIpolyi et. al., 2007).

Lastly, spatial memory decline has been shown in DAT patients. Carlesimo et al. (1994) show that DAT patients perform significantly worse on a forward and backward spatial memory span task than controls. Similarly, deIpolyi et al. (2007) report that DAT patients perform poorly on forward and backward route learning tests.

To summarize findings concerning DAT patients and their spatial skills, Monacelli et al. (2003, p. 1496) state: "Therefore, the deficits that define AD [Alzheimer's Disease] limit these patients to spatial orientation strategies that are based on visual perceptual analyses. In contrast, normal subjects can use orientation strategies that place greater emphasis on memory and verbal mechanisms.... This is consistent with evidence that separate spatial and verbal capacities are combined to influence orientation and navigation." This provides the starting point of my work to investigate the 'separate spatial and verbal capacities'. I hypothesize that the inability of DAT patients to perform spatial tasks on the same level as healthy control participant results in the former providing less spatial information in spontaneous narratives. While this research is not claiming that providing spatial information in narratives is dependent on spatial navigation skills, it based on the idea that if spatial orientation and navigation is a difficult task for DAT patients, they will not voluntarily provide this kind of information in an unguided task. My research is thus only a starting point into if/how spatial reasoning and verbal performance are connected in DAT patients.

2.3 DAT AND LANGUAGE. There is broad consensus in the literature that DAT patients' linguistic abilities deteriorate, often before the DAT diagnosis is made (Verma \& Howard, 2012). Consequently, linguistic deterioration is often seen as a diagnostic marker of DAT (Coughlan et al., 2018, among others).

To identify the precise linguistic impairment and potentially aid the diagnosis of DAT, different linguistic abilities of DAT patients have been investigated. Overall these studies indicate that early on the patients' performance on semantic tasks, such as naming and wordfinding, deteriorates; other linguistic abilities, such as pronunciation and syntax, remain intact in the early stages of the disease but are affected later on as well (Fraser et al., 2016; Forbes et al., 2002). Building on such findings, Orimaye et al. (2017) describe a variety of syntactic and semantic features that differ significantly between DAT patients and a control group. The identified significant differences include the number of predicates included in utterances, the frequency of repetitions, and the inclusion of word replacements and incomplete words. In addition, these researchers also found several linguistic features that did not differ between DAT 
and healthy participants, including the use of coordinated and subordinated sentences, the inclusion of function words, the overall word count, and the number of morphemes.

Two aspects of the linguistic performance of DAT patients seem particularly relevant for the current work, namely the use of preposition as well as the patients' precise semantic impairments. In terms of word classes, Thomas et al. (2005) found that "closed class words were particularly helpful in predicting the level of language deficit in patients" (p. 1754). Furthermore, Bucks et al. (2000) observed that DAT patients use pronouns, adjectives, and verbs more than control participants, but the DAT patients use fewer nouns. Similarly, Alegria et al. (2013) show that prepositions, pronouns, adverbs, and interjections are used more by DAT patients than by healthy controls. Additionally, Fraser et al. (2016) found that DAT patients produced significantly more pronouns and high frequency words, while producing fewer nouns than controls. This finding leads to their suggestion that " $\mathrm{t}]$ his potential connection between the omission of certain content words [nouns by DAT patients] and a reduction in prepositional phrases will require further investigation in future work." (pp. 415-416) This is directly addressed by my research question: do DAT patients spontaneously include fewer spatial prepositions (and therefore prepositional phrases) than healthy controls in narratives?

Turning to the actual semantic content provided by DAT patients, Thomas et al. (2005) describe the linguistic deterioration in DAT patients to include "shallow vocabulary" (p. 1570). According to Fraser et al. (2016, p. 414), the semantic information provided by DAT patients is "relatively uninformative [in] picture descriptions". Beyond these broad generalizations, few studies have investigated actual semantic content that is missing from DAT patients' narratives. Mostly naming studies have been performed to investigate the semantic knowledge of DAT patients. These typically show impairments for DAT participants on such tasks (see Verma and Howard (2012) among others). Given this lack of detailed investigations into the type of semantic content missing from DAT patients' language, I investigate whether specifically spatial information is provided at a different frequency by DAT and control participants. The idea is that DAT patients' inability to navigate space effectively is reflected in them not providing information about spatial relations in spontaneous speech.

\section{Materials and Method.}

3.1 PiCTURE NARRATIVE. According to Forbes et al. (2002), "[v]erbal picture descriptions are among the most sensitive measures for assessing spontaneous speech in AD." (p. 357) This is due to the fact that the presence of the picture reduces the memory load. Furthermore, it allows the participants to freely decide on the aspects of the picture they want to talk about. Consequently, I picked picture narratives for this analysis.

3.2 PARTICIPANTS. 226 transcripts of the Boston Cookie Theft picture narratives were selected from the Pitt Corpus (Becker et al., 1994) available at DementiaBank. ${ }^{3}$ The transcripts represented 89 healthy individuals and 137 individuals classified as 'dementia probable' (DAT). For those individuals who had more than one transcript of the cookie theft picture available in the database, the latest available recording was chosen. Consequently, the age of the participants is not always precisely known, but with that caveat in mind, the healthy adults ranged from 4681 years and the dementia patients were 53-88 years of age. 53 healthy participants were female; 90 of DAT participants identified as female.

\footnotetext{
${ }^{3}$ Orimaye et al. (2017) states that this picture "has been shown to be clinically relevant in identifying linguistic deficits in both Alzheimer's disease and Aphasia patients." (p. 3)
} 
3.3 METHOD. Using CLAN, all utterances including the terms on, in, at, from, to, into, onto, left, and right were extracted from the transcripts. ${ }^{4}$ All of these utterances were coded by hand for their spatial use of the relevant term. Immediate word repetitions were excluded (as to not skew the quantitative analysis). Furthermore, all uses of these terms referring to clothes and glasses were excluded (e.g. she has a dress on, she's in a dress, she has glasses on) although these uses could arguably be viewed as including spatial information (e.g. the dress is 'located' on the girl). The frequency of the use of each spatial term was recorded for each participant. The frequencies were then normed to 100 words using each participant's word count (ranging from 32-485 words for healthy participants and 22-300 for DAT patients). Due to the fact that the normality assumption was not met, the non-parametric Mann-Whitney U test was performed for all statistical analyses.

\section{Results.}

4.1 LEFT VS. RIGHT. The first analysis looked at the use of the inclusion of the spatial terms left and right. The normed frequencies of the two terms were combined due to the overall low number of occurrences of each term. The Mann-Whitney U test returned a significant result $(\mathrm{Z}(137,89)=-2.946, \mathrm{p}=.003)$, indicating that the healthy and DAT participants did not include this information equally often in their descriptions; the healthy participants (Mean $=.21$, Median $=0$ ) provided this information more often than the DAT participants (Mean $=.03$, Median $=0$ )

4.2 LOCATION. The next analysis performed combined the normed frequencies of three spatial terms often used to indicate location (on, in, at) in order to see if the healthy and DAT participants included this information equally often. No significant differences were found concerning the inclusion of information about the location by DAT and control participants $(\mathrm{Z}(13789)=-.14, \mathrm{p}>.05)$.

4.3 DiRECTION. Next, the information concerning direction was investigated. For this, the normed frequencies of four terms often used to indicate direction (from, to, into, onto) were combined. A significant difference between the control group and the DAT patients was observed $(\mathrm{Z}(137,89)--3.447, \mathrm{p}=.001)$. Control participants $($ Mean $=.921$, Median $=.847)$ provided directional information more frequently than DAT participants $($ Mean $=.59$, Median $=$ $0)$.

4.4 STATIC AND DYNAMIC. To follow up on the results for the information concerning location and direction, some data was recoded to perform a more stringent analysis of this information. During the spatial coding of the words on and in, it was noticed that sometimes participants used these terms with a directional meaning (e.g. the water spilled on the floor) rather than a strictly locational one. Consequently, the data for in, on, into and onto was recoded for this directional use, and two new groups of words were formed. Group 1 included the uses of in, on, and at representing truly static, locational information; group 2 was created by combining the uses of in and on with into, onto, from and to to investigate the truly dynamic, directional information provided by the participants. The analyses for these new groups of spatial information confirm the previous findings: the static information is included equally often by healthy and DAT participants $(Z(137,89)=-.075, \mathrm{p}=.94)$ whereas the inclusion of dynamic information shows a

\footnotetext{
${ }^{4}$ The cardinal directions were not included as they are typically not used for small scale description, such as the cookie theft picture, in English. Furthermore, the verbs carrying spatial information were left for future research (see discussion).
} 
significant difference between the groups $(Z(137,89)=-2.299, p=.022)$. The control group $($ Mean $=1.16$, Median $=.935)$ includes dynamic information more often than the DAT participants $($ Mean $=.911$, Median $=0)$.

5. Discussion. The finding concerning the equal inclusion of left and right by DAT patients and healthy controls supports the findings by Flicker et al. (1988) who observed that DAT patients performed worse on a left/right road map test than control participants. However, neither Brouwers et al. (1984) nor Cherrier et al. (2001) observed such a significant difference between DAT and control participants, indicating that the left/right orientation skills in DAT patients require further investigation. While my finding indicates that there is a difference in the usage of these terms, the overall usage of these terms in the narratives was very low (healthy: $.25 \%$ of all words with only 9/89 participants using one or both of the terms; DAT: $.06 \%$ of all words with only 2/137 participants using one or both terms), likely due to the fact that this information is not central to the depicted scene (i.e. it's not important whether the mother's left or right foot is in the puddle or whether the boy uses his left or right hand to get a cookie out of the jar). This overall minimal use of these terms might have influenced my significant finding. To ensure that these are robust findings, the production and comprehension of these terms as well as their applicability to real-world navigation and orientation tasks should be empirically investigated further. As pointed out by Fraser et al. (2016) the short length of the picture descriptions might also not be sufficiently appropriate for this kind of corpus analysis.

The findings concerning the different use of locational/static and directional/dynamic prepositions were unexpected and new. To my knowledge, no such difference with respect to these spatial terms and concepts has been reported for DAT patients. Yet, these findings can be connected to the observations about the spatial domain in two ways: the difference between routes and landmarks in navigation tasks, and the relational framework of spatial terms.

5.1 LANDMARKS AND ROUTES. Many researchers that have tested DAT patients on their route learning abilities include a 'landmark' test. During the learning phase of a novel route, different landmarks (usually 5-10) are pointed out to the participants by the experimenters. Out of all the aspects tested by the experimenters in the route learning tasks, DAT patients seem to perform best on tests related to the landmarks (and consistently perform worse on the actual navigation and locating tasks). deIpolyi et al. (2007) report that DAT participants performed equally well to healthy controls on a landmark recognition task in which they were shown photographs of the landmarks and had to indicate whether the landmark was seen on the route. This performance of DAT patients could be seen as comparable to the inclusion of locative/static spatial information as the landmarks are static. In contrast, the DAT patients perform worse when asked to recall the learned route correctly which could be comparable to the inclusion of directional/dynamic information in narratives. However, these findings are not fully consistent with those reported by Cherrier et al. (2001) who found significant differences between DAT patients and healthy participants on such a landmark recognition task. Thus, it seems worthwhile to further investigate the static/locative information that DAT patients can recognize, comprehend and produce correctly.

5.2 RELATIONAL FRAMEWORK FOR SPATIAL MEANING. The significant differences between static and dynamic spatial prepositions can be aligned with Chatterjee's (2008) observations about the semantics of spatial terms. He divides the semantic aspects of spatial language into four components along two dimensions (static/dynamic and intrinsic/extrinsic), as shown in table 1. The intrinsic dimension refers to properties of the object, while the extrinsic dimension puts the 
object into a relation with another object. The static dimension excludes movement whereas the dynamic classification requires movement.

\begin{tabular}{lll} 
& Intrinsic & Extrinsic \\
\hline Static & Object, proper & Locative relations \\
\hline Dynamic & Manner of motion & Path of motion \\
& Table 1: Semantic Framework
\end{tabular}

The four possible categories are consequently 'static and intrinsic' which are properties of the object itself (e.g. color, size, texture). The category 'static and extrinsic' describes the object in a static relation with another object (e.g. the first object is on/in/under the second object). The category 'dynamic and intrinsic' refers to movement the object can perform itself (e.g. rotate, roll). Lastly, 'dynamic and extrinsic' describes movement of the object along a path and therefore in relation to the world/ another object (e.g. the first object is going towards/into/onto the second object). As can be seen from these explanations, the extrinsic categories reflect the different spatial prepositions of English. In relation to my findings, this means that the DAT patients show a stronger deterioration in the 'dynamic and extrinsic' category than in the 'static and extrinsic' category. This warrants further investigation into the patterns of spatial language deterioration of DAT patients. One could expect a similar pattern of deterioration for the intrinsic categories, but reflected in different word categories in English. The 'intrinsic and static' category of object would likely include nouns, whereas the 'dynamic and intrinsic' category would be represented by verbs (Chatterjee, 2008). However, that prediction of a similar split in the intrinsic category as observed for the extrinsic category seems to go against previous findings that DAT patients use markedly fewer nouns than healthy controls and more verbs (Bucks et al., 2000; Fraser et al., 2016). Neither offers detailed analysis into the semantics of the words in the categories they analyzed; thus, especially for the verbs it is possible a detailed analysis of the spatial and non-spatial verbs could provide more detailed insights. The current trend of 'dynamic and intrinsic' as well as 'static and extrinsic' being preserved the most in DAT patients seems puzzling.

A more detailed investigation of the usage of verbs by DAT patients could also investigate the variability of linguistic deterioration further. The findings concerning left/right - terms used by only two DAT patients - as well as previous work (Fraser et al., 2014, among others) indicate that the linguistic behavior of DAT patients could benefit from a more fine-grained investigation. This also includes the possible effects of the age of onset and sex as variables affecting the linguistic deterioration as Bucks et al. (2000) have suggested.

6. Conclusion. Dementia of the Alzheimer's type is initially characterized by the inability of the patients to navigate space effectively and a deterioration of the language skills. In this work, I have shown through a corpus study that the spatial navigation deficit is reflected in the spatial language production of DAT patients in narratives: While they perform equally well to healthy participants when it comes to static and locative information, DAT patient provide significantly less dynamic and directional information. Furthermore, they also use the terms left and right less in their spontaneous description of the cookie theft picture. Despite the limitations of this research, it makes one very important contribution: it establishes that DAT patients show different command of static and dynamic spatial language. This difference had not been observed before and calls for more detailed investigation of the production and comprehension of these terms as well as whether such performance is linked to the spatial navigation skills of these 
patients to see if these two cognitive skills are independently affected or deteriorate alongside one another.

\section{References}

Alegria, Renne, Celia Gallo, Mirian Bolso, Bernado dos Santos, Cleide Rosana Prisco, Cassino Bottino \& Nogeira Ines. 2013. Comparative study of the uses of grammatical categories: Adjectives, adverbs, pronouns, interjections, conjunctions and prepositions in patients with Alzheimer's disease. Alzheimer's \& Dementia: The Journal of the Alzheimer's Association 9(4). P882. https://doi.org/10.1016/j.jalz.2013.08.233.

Becker, James, Francois Boller, Oscar Lopez, Judith Saxton \& Karen McGonigle. 1994. The natural history of Alzheimer's disease. Archive of Neurology 51(6). 585-594.

Brouwers, Pim, Chris Cox, Alex Martin, Thomas Chase \& Paul Fedio. 1984. Differential perceptual-spatial impairment in Huntington's and Alzheimer's dementias. Archives of Neurology 41(10). 1073-1076. https://doi.org/10.1001/archneur.1984.04050210071017.

Bucks, Romola, S. Singh, J. Cuerden \& G. Wilcock. 2000. Analysis of spontaneous conversational speech in dementia of Alzheimer type: Evaluation of an objective technique for analysing lexical performance. Aphasiology 14(1). 71-91. https://doi.org/10.1080/026870300401603.

Carlesimo, Giovanni, Lucia Fadda, Sebastiano Lorusso \& Carlo Caltagirone. 1994. Verbal and spatial memory spans in Alzheimer's and multi-infarct dementia. Acta Neurologica Scandinavica 89(2). 132-138.

Chatterjee, Anjan. 2008. The neural organization of spatial thought and language. Seminars in Speech and Language 29(3). 226-238. https://doi.org/10.1055/s-0028-1082886.

Cherrier, Monique, Mario Mendez \& Kent Perryman. 2001. Route learning performance in Alzheimer disease patients. Neuropsychiatry, Neuropsychology, and Behavioral Neurology 14(3). 159-168.

Coughlan, Gillian, Jan Laczó, Jakub Hort, Anne-Marie Minihane \& Michael Hornberger. 2018. Spatial navigation deficits - overlooked cognitive marker for preclinical Alzheimer disease?. Nature Reviews Neurology 14. 496-506.

deIpolyi, Amy, Katherine Rankin, Lennart Mucke, Bruce Miller \& Maria Gorno-Tempini. 2007. Spatial cognition and the human navigation network in AD and MCI. Neurology 69(10). 986-997. https://doi.org/10.1212/01.wn1.0000271376.19515.c6.

Flicker, Charles, Steven Ferris, Thomas Crook, Barry Reisberg \& Raymond Bartus. 1988. Equivalent spatial-rotation deficits in normal aging and Alzheimer's disease. Journal of Clinincal and Experimental Neuropsychology 10(4). 387-399. https://doi.org/10.1080/01688638808408247.

Forbes, Katrina, Annalena Venneri \& Michael Shanks. 2002. Distinct patterns of spontaneous speech deterioration: An early predictor of Alzheimer's disease. Brain and Cognition 48(23). 356-361.

Fraser, Kathleen, Jed Meltzer \& Frank Rudzicz. 2016. Linguistic features identify Alzheimer's disease in narrative speech. Journal of Alzheimer's Disease 49. 407-422. https://doi.org/10.3233/JAD-150520.

Fraser, Kathleen, Jed Meltzer, Laurie Graham, Carol Leonard, Graeme Hirst, Sandra Black \& Elizabeth Rochon. 2014. Automated classification of primary progressive aphasia subtypes from narrative speech transcripts. Cortex 55. 43-60. https://doi.org.10.1016/j.cortex.2012.12.006. 
Hermer-Vazquez, Linda, Anne Moffet \& Paul Munkholm. 2001. Language, space, and the development of cognitive flexibility in humans: The case of two spatial memory tasks. Cognition 79(3). 263-299.

Kemmerer, David. 2005. The spatial and temporal meanings of English prepositions can be independently impaired. Neuropsychologia 43(5). 797-806.

Landau, Barbara, Banchiamlack Dessalegn \& Ariel Goldberg. 2009. Language and space: Momentary interactions. Chilton, Paul \& Vyvyan Evans (eds.), Language, cognition and space: The state of the art and new directions. 51-78. London: Equinox Publishing.

Lithfous, Segolene, Andre Dufour \& Olivier Despres. 2013. Spatial Navigation in normal aging and the prodromal stage of Alzheimer's disease: Insights from imaging and behavioral studies. Ageing Research Reviews 12(1). 201-213. https://doi.org/10.1016/j.arr.2012.04.007.

Monacelli, Anthony, Laura Cushman, Voyko Kavcic \& Charles Duffy. 2003. Spatial disorientation in Alzheimer's disease. Neurology 61(11). 1491-1497.

Munnich, Edward, Barbara Landau \& Barbara Dosher. 2001. Spatial language and spatial representation: A cross-linguistic comparison. Cognition 81(3). 171-207.

Orimaye, Sylvester, Jojo Wong, Karen Golden, Chee Wong \& Ireneous Soyiri. 2017. Predicting probable Alzheimer's disesase using linguistic deficits and biomarkers. BMC Bioinformatics 18(1). 34. https://doi.org/10.1186/s12859-016-1456-0.

Thomas, Calvin, Vlado Keselj, Nick Cercone, Kenneth Rockwood \& Elissa Asp. 2005. Automatic detection and rating of dementia of Alzheimer type through lexical analysis of spontaneous speech. IEEE International Conference on Mechatronics \& Automation 3. 1569-1574. https://doi.org/10.1109/ICMA.2005.1626789.

Tillas, Alexandros. 2015. Language as grist to the mill of cognition. Cognitive Processing 16(3). 219-243. https://doi.org/10.1007/s10339-015-0656-2.

Verma, M. \& Robert Howard. 2012. Semantic memory and language dysfunction in early Alzheimer's disease: A review. International Journal of Geriatric Psychiatry 27(12). 1209-1217. https://doi.org/10.1002/gps.3766. 